\title{
How One can Repair Non-integrable Kahan Discretizations. II. A Planar System with Invariant Curves of Degree 6
}

\author{
Misha Schmalian $^{1} \cdot$ Yuri B. Suris $^{2}$ (D) Yuriy Tumarkin ${ }^{1}$ \\ Received: 7 July 2021 / Accepted: 28 October 2021 / Published online: 28 November 2021 \\ (c) The Author(s) 2021
}

\begin{abstract}
We find a novel one-parameter family of integrable quadratic Cremona maps of the plane preserving a pencil of curves of degree 6 and of genus 1 . They turn out to serve as Kahan-type discretizations of a novel family of quadratic vector fields possessing a polynomial integral of degree 6 whose level curves are of genus 1, as well. These vector fields are non-homogeneous generalizations of reduced Nahm systems for magnetic monopoles with icosahedral symmetry, introduced by Hitchin, Manton and Murray. The straightforward Kahan discretization of these novel non-homogeneous systems is non-integrable. However, this drawback is repaired by introducing adjustments of order $O\left(\epsilon^{2}\right)$ in the coefficients of the discretization, where $\epsilon$ is the stepsize.
\end{abstract}

Keywords Birational maps · Discrete integrable systems · Elliptic pencil - Rational elliptic surface $\cdot$ Integrable discretization

\section{Introduction}

The problem of integrable discretization [37] consists of finding, for a given integrable system, a discretization which remains integrable. All conventional general-purpose discretization methods for ODEs, like Runge-Kutta methods etc., applicable to arbi-

Communicated by F. W. Nijhoff.

$凶 \quad$ Yuri B. Suris

suris@math.tu-berlin.de

Misha Schmalian

mjs297@cam.ac.uk

Yuriy Tumarkin

yt354@cam.ac.uk

1 Trinity College, Trinity Street, Cambridge CB21TQ, UK

2 Institut für Mathematik, Technische Universität Berlin, MA 7-1, Str. des 17. Juni 136, 10623 Berlin, Germany 
trary ODEs, fail to preserve integrability when applied to a general integrable system. However, there exist "unconventional" numerical methods applicable to certain particular classes of ODEs which possess, on these classes, special properties, see [22]. One of such "unconventional" methods, known alternatively as Hirota-Kimura discretization or Kahan discretization, attracted recently much attention. It is applicable to any ODE on $\mathbb{R}^{n}$ with a quadratic vector field, and possesses remarkable properties concerning preservation of integrals and integral invariants. Consider a quadratic ODE

$$
\dot{x}=Q(x)+B x+c,
$$

where $Q: \mathbb{R}^{n} \rightarrow \mathbb{R}^{n}$ is a vector of quadratic forms, $B$ is an $n \times n$ matrix, and $c \in \mathbb{R}^{n}$. The Hirota-Kimura discretization method, or Kahan's discretization method, consists in replacing the time derivative on the left-hand side by the first difference of the numerical approximation $x: \in \mathbb{Z} \rightarrow \mathbb{R}^{n}$, while the quadratic expressions on the right-hand side are replaced by symmetric bilinear expressions in terms of $x=x(t)$ and $\tilde{x}=x(t+\epsilon)$ :

$$
\frac{\tilde{x}-x}{\epsilon}=Q(x, \tilde{x})+\frac{1}{2} B(x+\tilde{x})+c,
$$

where

$$
Q(x, \tilde{x})=\frac{1}{2}(Q(x+\tilde{x})-Q(x)-Q(\tilde{x}))
$$

is the symmetric bilinear form corresponding to the quadratic form $Q$. Equation (2) is linear with respect to $\tilde{x}$ and therefore defines a rational map $\tilde{x}=f(x, \epsilon)$. Due to the symmetry of equation (2) with respect to interchanging $x \leftrightarrow \tilde{x}$ accompanied by sign inversion $\epsilon \mapsto-\epsilon$, the map $f$ is reversible:

$$
f^{-1}(x, \epsilon)=f(x,-\epsilon)
$$

(in numerical analysis, discretization schemes with this property are called selfadjoint). Thus, the map $f$ is birational.

The origin of this discretization method can be seen in the pioneering work of Hirota in 1970s. One of his main contributions to the theory of solitons is an ingenious transformation of the majority, if not all, integrable PDEs to the so called bilinear form (which turned out to have extremely deep relations to various branches of mathematics, especially to infinite dimensional Lie algebras and their representation theory). He then invented a bilinear discretization of integrable PDEs presented in the bilinear form [1115], culminating in the discovery of the so called Hirota bilinear equation which is one of the most fundamental integrable systems [16]. In the context of ODEs, the bilinear discretization was applied to the Euler top and to the Lagrange top by Hirota and Kimura [17,21], who observed that the resulting maps are integrable.

Independently and in the general framework of quadratic ODEs (not necessarily integrable), the bilinear discretization was introduced by Kahan [20]. For some reasons 
which remain not completely clarified up to now, Kahan's method tends to preserve integrals of motion and integral invariants much more often than any other known general purpose discretization scheme, which was confirmed by extensive studies, see [23-30,32,33,36,40] and [2,3,5,6,38,39]. Generalizations of Kahan's method for higher order ODEs and/or to polynomial vector fields of higher degree were studied in $[4,19]$. Integrability is preserved by Kahan's method in an amazing number of cases (but not always!). It seems reasonable to reserve the term "Hirota-Kimura method" for this method in the context of integrable discretization. In this article, we use the term "Kahan's method".

Simple counterexamples to preservation of integrability by Kahan's method are available already in dimension $n=2$, and can be found among non-homogeneous extensions of the so called reduced Nahm equations introduced in [18]. These are the systems of the form

$$
\left(\begin{array}{c}
\dot{x} \\
\dot{y}
\end{array}\right)=\frac{1}{\rho(x, y)}\left(\begin{array}{c}
\partial H / \partial y \\
-\partial H / \partial x
\end{array}\right),
$$

where

$$
H(x, y)=\ell_{1}^{\gamma_{1}}(x, y) \ell_{2}^{\gamma_{2}}(x, y) \ell_{3}^{\gamma_{3}}(x, y), \quad \rho(x, y)=\ell_{1}^{\gamma_{1}-1}(x, y) \ell_{2}^{\gamma_{2}-1}(x, y) \ell_{3}^{\gamma_{3}-1}(x, y),
$$

with $\gamma_{1}, \gamma_{2}, \gamma_{3} \in \mathbb{R} \backslash\{0\}$, and $\ell_{i}(x, y)=a_{i} x+b_{i} y$ are linear forms. Integrability takes place for $\left(\gamma_{1}, \gamma_{2}, \gamma_{3}\right)=(1,1,1),(1,1,2)$, and $(1,2,3)$. In all three cases, all integral curves of the system (4) are of genus 1 . In [5,24,32] integrability was established for the Kahan discretization of all three cases of the reduced Nahm equations.

If $\left(\gamma_{1}, \gamma_{2}, \gamma_{3}\right)=(1,1,1)$, one is dealing with a canonical Hamiltonian system with a homogeneous cubic Hamilton function. As discovered in [2], in this situation the Kahan discretization remains integrable for arbitrary (i.e., also for non-homogeneous) cubic Hamilton functions.

If $\left(\gamma_{1}, \gamma_{2}, \gamma_{3}\right)=(1,1,2)$, one can find non-homogeneous perturbations of the quartic polynomial $H(x, y)$ so that the resulting differential equations (4) still have the above mentioned property: all integral curves are of genus 1. A Kahan discretization of the perturbed (non-homogeneous) system is non-integrable. However, it was shown in [33] that one can adjust the coefficients of the discretization (making them dependent on $\epsilon$ in a non-trivial way) to obtain an integrable Kahan-type discretization.

The present paper is devoted to a similar result for systems of the class $\left(\gamma_{1}, \gamma_{2}, \gamma_{3}\right)=$ $(1,2,3)$. The homogeneous system can be taken as

$$
\left\{\begin{array}{l}
\dot{x}=-2 x^{2}+2 x y \\
\dot{y}=-y^{2}+2 x y
\end{array}\right.
$$

It possesses an integral of motion of degree 6:

$$
H(x, y)=x^{2} y^{3}\left(-\frac{2}{3} x+\frac{1}{2} y\right)
$$


whose level sets are curves of genus 1 . The Kahan discretization of this system reads:

$$
\left\{\begin{array}{l}
(\tilde{x}-x) / \epsilon=-2 \tilde{x} x+(\tilde{x} y+x \tilde{y}) \\
(\widetilde{y}-y) / \epsilon=-\tilde{y} y+(\tilde{x} y+x \widetilde{y})
\end{array}\right.
$$

It is integrable, with an integral of motion

$$
H_{1}(x, y)=\frac{H(x, y)}{\left(1-\epsilon^{2} x^{2}\right)\left(1-\epsilon^{2}(x-y)^{2}\right)\left(1-\epsilon^{2}\left(x^{2}+y^{2}\right)\right)} .
$$

Consider the following non-homogeneous perturbation of system (5):

$$
\left\{\begin{array}{l}
\dot{x}=-2 x^{2}+2 x y+c \\
\dot{y}=-y^{2}+2 x y
\end{array}\right.
$$

It has the following integral of motion:

$$
H(x, y)=(x y+c)^{2}\left(-\frac{2}{3} x y+\frac{1}{2} y^{2}+\frac{1}{3} c\right),
$$

with the same property as above (all level sets are curves of genus 1). The Kahan discretization of this system,

$$
\left\{\begin{array}{l}
(\tilde{x}-x) / \epsilon=-2 x \tilde{x}+(\tilde{x} y+x \tilde{y})+c \\
(\tilde{y}-y) / \epsilon=-y \tilde{y}+(\tilde{x} y+x \tilde{y})
\end{array}\right.
$$

generates a non-integrable map. However, the coefficients of this discretization can be adjusted via $O\left(\epsilon^{2}\right)$ terms, to produce an integrable map:

$$
\left\{\begin{array}{l}
(\tilde{x}-x) / \epsilon=-\left(2-\epsilon^{2} c\right) x \tilde{x}+\left(1+\epsilon^{2} c\right)(\tilde{x} y+x \widetilde{y})+c-\epsilon^{2} c\left(2+\epsilon^{2} c\right) y \tilde{y}, \\
(\tilde{y}-y) / \epsilon=-\left(1+\epsilon^{2} c\right) y \tilde{y}+(\widetilde{x} y+x \tilde{y}) .
\end{array}\right.
$$

This map, like the unperturbed one (7), has an integral of motion whose level sets are curves of degree 6 and of genus 1 (the irreducible ones).

The presentation is organized as follows. In Sect. 2, we consider in detail system (5) and its Kahan discretization (7), paying special attention to the singularity confinement property of the latter map. In Sect. 3, we perform, following [31], a reduction of the pencil of invariant curves of degree 6 of the Kahan discretization to a pencil of biquadratic curves. This way, the map is shown to be birationally equivalent to a special QRT root (cf. [10,35]). In Sect. 4, we show that the relevant geometric and dynamical properties of this QRT root can be found in a one-parameter family of such maps, and then find a corresponding one-parameter family of birationally equivalent Kahan-type maps preserving a pencil of curves of degree 6 and of genus 1. Finally, in Sect. 5, a continuous limit is performed in those Kahan-type maps, leading to a novel integrable system (9), with a pencil of invariant curves with the same property (level sets of the non-homogeneous sextic polynomial (10) are of genus 1). 


\section{A Homogeneous $(1,2,3)$ System and Its Kahan Discretization}

We start with a reduced Nahm system (5) with $\left(\gamma_{1}, \gamma_{2}, \gamma_{3}\right)=(1,2,3)$, obtained by the following choice of the corresponding linear forms:

$$
\ell_{1}(x, y)=-\frac{2}{3} x+\frac{1}{2} y, \quad \ell_{2}(x, y)=x, \quad \ell_{3}(x, y)=y,
$$

so that $H(x, y)$ is as given in (6), and $\rho(x, y)=x y^{2}$.

Its Kahan discretization is given in (7). Due to homogeneity, we can restrict ourselves to the case $\epsilon=1$,

$$
\left\{\begin{array}{l}
\tilde{x}-x=-2 \tilde{x} x+(\tilde{x} y+x \tilde{y}) \\
\tilde{y}-y=-\tilde{y} y+(\tilde{x} y+x \tilde{y})
\end{array}\right.
$$

The general case is obtained from this by the re-scaling $(x, y) \mapsto(\epsilon x, \epsilon y)$. A simple computation gives an explicit formula for the map $f$ :

$$
\left(\begin{array}{l}
\tilde{x} \\
\tilde{y}
\end{array}\right)=\left(\begin{array}{cc}
1+2 x-y & -x \\
-y & 1-x+y
\end{array}\right)^{-1}\left(\begin{array}{l}
x \\
y
\end{array}\right)=\frac{1}{\Delta}\left(\begin{array}{cc}
1-x+y & x \\
y & 1+2 x-y
\end{array}\right)\left(\begin{array}{l}
x \\
y
\end{array}\right)
$$

or

$$
\tilde{x}=\frac{x(1-x+2 y)}{\Delta}, \quad \tilde{y}=\frac{y(1+3 x-y)}{\Delta}, \quad \Delta=1+x-2 x^{2}+2 x y-y^{2} .
$$

In homogeneous coordinates,

$$
f(x, y, z)=\left[x(z-x+2 y): y(z+3 x-y): z^{2}+z x-2 x^{2}+2 x y-y^{2}\right] .
$$

In the following proposition, we collect the relevant information about this map, as found in $[5,24,32,40]$.

Proposition 1 The map $f$ given in (14) admits an integral of motion

$$
H_{1}(x, y)=\frac{H(x, y)}{\left(1-x^{2}\right)\left(1-(x-y)^{2}\right)\left(1-\left(x^{2}+y^{2}\right)\right)},
$$

with $H(x, y)=x^{2} y^{3}\left(-\frac{2}{3} x+\frac{1}{2} y\right)$. The pencil of the level curves $H_{1}(x, y)=\lambda$, i.e.,

$$
H(x, y)-\lambda\left(1-x^{2}\right)\left(1-(x-y)^{2}\right)\left(1-\left(x^{2}+y^{2}\right)\right)=0,
$$

of $\operatorname{deg}=6$ possesses eleven (distinct) base points given by: 
- six finite base points of multiplicity 1 on the line $\ell_{1}=0$ :

$$
p_{1}=-p_{6}=\left(\frac{3}{5}, \frac{4}{5}\right), \quad p_{2}=-p_{5}=\left(1, \frac{4}{3}\right), \quad p_{3}=-p_{4}=(3,4),
$$

- three base points of multiplicity 2 on the line $\ell_{2}=0$, two finite and one at infinity:

$$
p_{7}=-p_{9}=(0,-1), \quad p_{8}=[0: 1: 0],
$$

- and two finite base points of multiplicity 3 on the line $\ell_{3}=0$ :

$$
p_{10}=-p_{11}=(-1,0) \text {. }
$$

See Fig. 1 for an illustration. One has: $\mathcal{I}(f)=\left\{p_{6}, p_{9}, p_{11}\right\}$ and $\mathcal{I}\left(f^{-1}\right)=$ $\left\{p_{1}, p_{7}, p_{10}\right\}$. All base points participate in three confined singular orbits of the map $f:$

$$
\begin{aligned}
& \left(p_{9} p_{11}\right) \longrightarrow p_{1} \longrightarrow p_{2} \longrightarrow p_{3} \longrightarrow p_{4} \longrightarrow p_{5} \longrightarrow p_{6} \longrightarrow\left(p_{7} p_{10}\right), \\
& \left(p_{6} p_{11}\right) \longrightarrow p_{7} \longrightarrow p_{8} \longrightarrow p_{9} \longrightarrow\left(p_{1} p_{10}\right), \\
& \left(p_{6} p_{9}\right) \longrightarrow p_{10} \longrightarrow p_{11} \longrightarrow\left(p_{1} p_{7}\right)
\end{aligned}
$$

We refer the reader to [1,7-9] for general information about birational (Cremona) maps of $\mathbb{C P}^{2}$, including the notion of confined singular orbits (related to degreelowering curves and dynamical degree, or algebraic entropy).

\section{Reduction of the Map $f$ to a Special QRT Root}

We use notation

$$
\mathcal{E}_{6}=\mathcal{P}\left(6 ; p_{1}, \ldots, p_{6}, p_{7}^{2}, p_{8}^{2}, p_{9}^{2}, p_{10}^{3}, p_{11}^{3}\right)
$$

for the pencil of curves of degree 6 with simple base boints $p_{1}, \ldots, p_{6}$, double base points $p_{7}, p_{8}, p_{9}$, and triple base points $p_{10}, p_{11}$. One can simplify such a pencil by applying a quadratic Cremona map $\phi$ with the fundamental points $p_{9}, p_{10}, p_{11}$ (the both triple base points and one of the double base points), cf. [31].

Proposition 2 Consider a quadratic Cremona map $\phi$ blowing down the lines $\left(p_{10} p_{11}\right)$, ( $\left.p_{9} p_{11}\right),\left(p_{9} p_{10}\right)$ to points denoted by $q_{9}, q_{10}, q_{11}$, respectively, and blowing up the points $p_{9}, p_{10}, p_{11}$ to the lines $\left(q_{10} q_{11}\right),\left(q_{9} q_{11}\right),\left(q_{9} q_{10}\right)$. All other base points $p_{i}$, $i=1, \ldots, 8$ are regular points of $\phi$ and their images are denoted by $q_{i}=\phi\left(p_{i}\right)$. The change of variables $\phi$ maps pencil (22) of sextic curves to the pencil

$$
\mathcal{E}_{4}=\mathcal{P}\left(4 ; q_{1}, \ldots, q_{6}, q_{10}, q_{11}, q_{7}^{2}, q_{8}^{2}\right)
$$

of quartic curves with eight simple base points and two double base points. The point $q_{9}$ is not a base point of the latter pencil. 


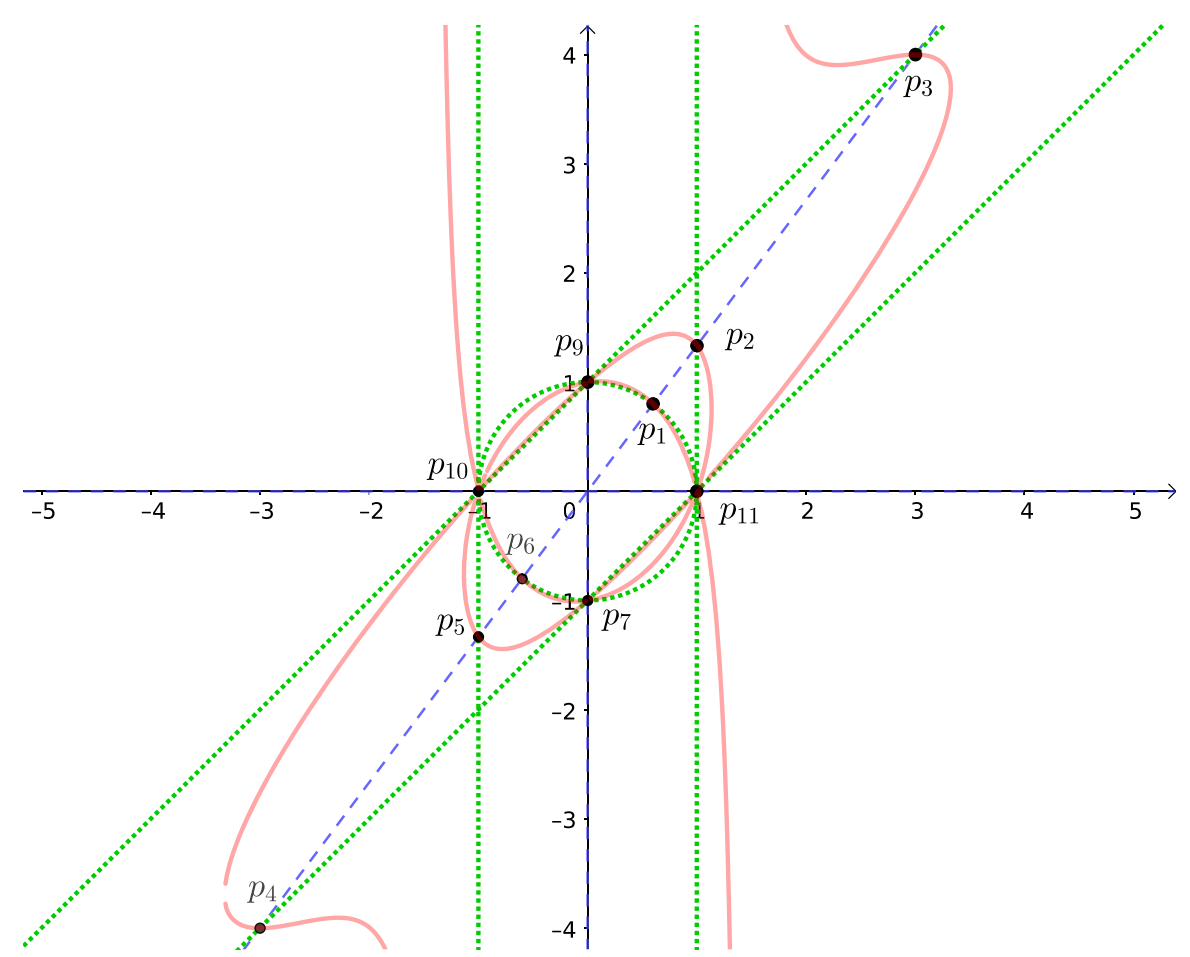

Fig. 1 Some invariant curves of the sextic pencil (17). Blue: three lines (one of them double and one triple)— the vanishing set of the numerator of $H_{1}(x, y)$, i.e., $\lambda=0$. Green: four lines and a conic-the vanishing set of the denominator of $H_{1}(x, y)$, i.e., $\lambda=\infty$. Red: a generic curve

Proof The total image of a curve $C \in \mathcal{E}_{6}$ is a curve of degree 12. Since $C$ passes through $p_{9}, p_{10}, p_{11}$ with multiplicities $2,3,3$, its total image contains the lines $\left(q_{10} q_{11}\right)$, $\left(q_{9} q_{11}\right),\left(q_{9} q_{10}\right)$ with the same multiplicities. Dividing by the linear defining polynomials of all these lines, we see that the proper image of $C$ is a curve of degree $12-8=4$. This curve passes through all points $q_{i}, i=1, \ldots, 8$ (for $i=7,8$ with multiplicity 2). The curve $C$ of degree 6 has no other intersections with the line $\left(p_{10} p_{11}\right)$ different from two triple points $p_{10}$ and $p_{11}$, therefore its proper image does not pass through $q_{9}$. On the other hand, the curve $C$ of degree 6 has one additional intersection point with each of the lines $\left(p_{9} p_{10}\right)$ and $\left(p_{9} p_{11}\right)$, different from the double point $p_{9}$ and the triple point $p_{10}$, respectively $p_{11}$. Therefore, its proper image passes through $q_{11}$, resp. $q_{10}$, with multiplicity 1 .

For the proof of the following Proposition, we will repeatedly use the following lemma.

Lemma 3 Let $F$ be a quadratic Cremona map with $\mathcal{I}(F)=\{a, b, c\}$, and let $F$ blow down the lines $(a b),(b c)$, ( $c a)$ to the points $C, A, B$, respectively. Then the image of a generic line under $F$ is a conic through $A, B, C$. The (proper) image of a line through one of the indeterminacy points, say of the line $(a d)$, is the line $(A D)$, where $D=F(d)$. 
Proof The total image of the line $(a d)$ is a conic, but since $a$ is blown up to a line, the proper image is a line. This line has to pass through $D=F(d)$ and through $A$ (since the line $(a d)$ intersects the line $(b c)$ which is blown down to $A)$.

Proposition 4 The map $g=\phi \circ f \circ \phi^{-1}$ has three confined singular orbits:

$$
\begin{aligned}
& \left(q_{6} q_{8}\right) \longrightarrow q_{10} \longrightarrow q_{1} \longrightarrow q_{2} \longrightarrow q_{3} \longrightarrow q_{4} \longrightarrow q_{5} \longrightarrow q_{6} \longrightarrow\left(q_{7} q_{10}\right) \\
& \left(q_{6} q_{11}\right) \longrightarrow q_{7} \longrightarrow q_{8} \longrightarrow\left(q_{10} q_{11}\right) \\
& \left(q_{8} q_{11}\right) \longrightarrow q_{11} \longrightarrow\left(q_{7} q_{11}\right)
\end{aligned}
$$

The point $q_{9}$ is its fixed point, and lies on the line $\left(q_{7} q_{8}\right)$. Moreover, the points $q_{3}$ and $q_{11}$ are infinitely near.

Proof We have:

$$
q_{i} \stackrel{\phi^{-1}}{\longrightarrow} p_{i} \stackrel{f}{\longrightarrow} p_{i+1} \stackrel{\phi}{\longrightarrow} q_{i+1}, \quad i=1, \ldots, 5 .
$$

Further,

$$
q_{6} \stackrel{\phi^{-1}}{\longrightarrow} p_{6} \stackrel{f}{\longrightarrow}\left(p_{7} p_{10}\right) \stackrel{\phi}{\longrightarrow}\left(q_{7} q_{10}\right)
$$

(applying Lemma 3 for $\phi$ );

$$
\begin{aligned}
& q_{7} \stackrel{\phi^{-1}}{\longrightarrow} p_{7} \stackrel{f}{\longrightarrow} p_{8} \stackrel{\phi}{\longrightarrow} q_{8} ; \\
& q_{8} \stackrel{\phi^{-1}}{\longrightarrow} p_{8} \stackrel{f}{\longrightarrow} p_{9} \stackrel{\phi}{\longrightarrow}\left(q_{10} q_{11}\right) ; \\
& q_{9} \stackrel{\phi^{-1}}{\longrightarrow}\left(p_{10} p_{11}\right) \stackrel{f}{\longrightarrow}\left(p_{10} p_{11}\right) \stackrel{\phi}{\longrightarrow} q_{9}
\end{aligned}
$$

(applying Lemma 3 for $f$ );

$$
\begin{aligned}
& q_{10} \stackrel{\phi^{-1}}{\longrightarrow}\left(p_{9} p_{11}\right) \stackrel{f}{\longrightarrow} p_{1} \stackrel{\phi}{\longrightarrow} q_{1} ; \\
& q_{11} \stackrel{\phi^{-1}}{\longrightarrow}\left(p_{9} p_{10}\right) \stackrel{f}{\longrightarrow}\left(p_{7} p_{11}\right) \stackrel{\phi}{\longrightarrow}\left(q_{7} q_{11}\right)
\end{aligned}
$$

(applying Lemma 3 for $f$, then for $\phi$ ).

Next, we consider lines which are blown down by $g$ :

$$
\left(q_{6} q_{8}\right) \stackrel{\phi^{-1}}{\longrightarrow} C\left(p_{6}, p_{8}, p_{9}, p_{10}, p_{11}\right) \stackrel{f}{\longrightarrow}\left(p_{9} p_{11}\right) \stackrel{\phi}{\longrightarrow} q_{10}
$$

(indeed, the total $f$-image of the conic is a curve of degree 4; however, three lines split off, being the blow-ups of $p_{6}, p_{9}, p_{11}$; thus, the proper image is the line through $f\left(p_{8}\right)=p_{9}$ and $\left.f\left(p_{10}\right)=p_{11}\right)$;

$$
\left(q_{6} q_{11}\right) \stackrel{\phi^{-1}}{\longrightarrow}\left(p_{6} p_{11}\right) \stackrel{f}{\longrightarrow} p_{7} \stackrel{\phi}{\longrightarrow} q_{7}
$$


(applying Lemma 3 for $\phi^{-1}$ );

$$
\left(q_{8} q_{11}\right) \stackrel{\phi^{-1}}{\longrightarrow}\left(p_{8} p_{11}\right) \stackrel{f}{\longrightarrow}\left(p_{9} p_{10}\right) \stackrel{\phi}{\longrightarrow} q_{11}
$$

(applying Lemma 3 for $\phi^{-1}$, then for $f$ ).

The fact that $q_{3}$ and $q_{11}$ are infinitely near follows from the fact that $p_{3} \in\left(p_{9} p_{10}\right)$, the latter line being blown down to $q_{11}$ by $\phi$.

It remains to show that $q_{9} \in\left(q_{7} q_{8}\right)$. For this, observe that the total $\phi$-image of $\left(p_{7} p_{8}\right)$ is the conic $C\left(q_{7}, q_{8}, q_{9}, q_{10}, q_{11}\right)$. However, since $p_{9} \in\left(p_{7} p_{8}\right)$, the blow-up of $p_{9}$ splits off this conic. This is the line $\left(q_{10} q_{11}\right)$, and it does not contain any of the points $q_{7}, q_{8}, q_{9}$. Thus, the proper $\phi$-image of ( $\left.p_{7} p_{8}\right)$ is a line containing the latter three points, which are therefore collinear.

For an actual computation of the map $\phi$, we can assume, without loss of generality, that pencil (23) consists of symmetric biquadratics, i.e., its double points are

$$
q_{7}=\phi\left(p_{7}\right)=[0: 1: 0], \quad q_{8}=\phi\left(p_{8}\right)=[1: 0: 0],
$$

while the points

$$
q_{9}=\phi\left(\left(p_{10} p_{11}\right)\right)=\phi(\{y=0\}), \quad q_{11}=\phi\left(\left(p_{9} p_{10}\right)\right)=\phi(\{y-x=1\})
$$

lie on the symmetry axis $u=v$. This still leaves us with one free parameter. It can be chosen so that

$$
\phi(x, y, z)=\left[z^{2}-x^{2}-y^{2}+x y: z^{2}-x^{2}-y z: x y\right] .
$$

A direct computation with this formula gives:

$$
\begin{aligned}
q_{1} & =\left(1,-\frac{1}{3}\right), \quad q_{2}=\left(-\frac{1}{3},-1\right), \quad q_{4}=\left(-1,-\frac{1}{3}\right), \quad q_{5}=\left(-\frac{1}{3}, 1\right), \\
q_{6} & =(1,3), \quad q_{7}=[0: 1: 0], \quad q_{8}=[1: 0: 0], \quad q_{9}=[1: 1: 0], \quad q_{10}=(3,1), \\
q_{11} & =(-1,-1), \quad q_{3}>q_{11}(\text { slope }-1) .
\end{aligned}
$$

The latter notation means that the point $q_{3}$ is infinitely near to $q_{11}$ and corresponds to the tangent line $\{v=-u-2\}$ there.

Now it remains to compute the map $g=\phi^{-1} \circ f \circ \phi$, i.e., the map $f$ in the coordinates $[u: v: w]$. A direct computation shows that, in the non-homogeneous coordinates, $g(u, v, 1)=[\tilde{u}: \widetilde{v}: 1]$ with

$$
\tilde{u}=v, \quad \widetilde{v}=\frac{u v-u-2}{2 u-v+1},
$$

and admits an integral of motion

$$
K(u, v)=\frac{3(u-v)^{2}-2(u+v)-4}{\left(u^{2}-1\right)\left(v^{2}-1\right)} .
$$




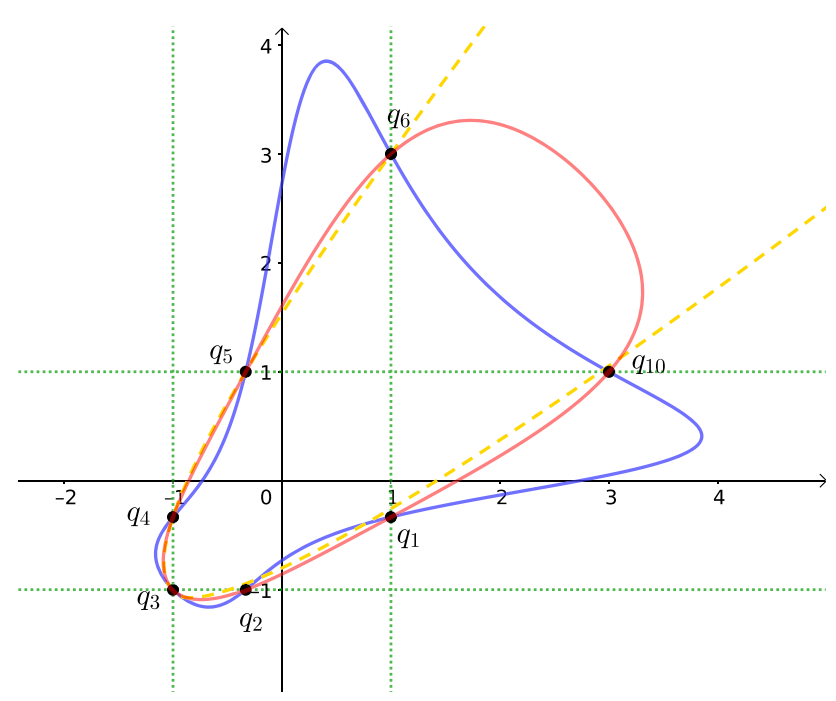

Fig. 2 Two biquadratics of the pencil $\{K(u, v)=\lambda\}$ with $K(u, v)$ from (27). All biquadratics pass through $q_{11}=(-1,-1)$ with the slope -1 (corresponding to $\left.q_{3}\right)$. Yellow: the conic through the eight base points $q_{1}, \ldots, q_{6}, q_{10}, q_{11}$. Green: four lines $\{u= \pm 1\},\{v= \pm 1\}$

Thus, all base points lie on the four lines $\{u= \pm 1\},\{v= \pm 1\}$, while the eight finite base points $q_{1}, \ldots, q_{6}, q_{10}, q_{11}$ lie on the conic (parabola) $\left\{3(u-v)^{2}-2(u+v)-4=\right.$ 0\}. See Fig. 2.

\section{Generalization of the QRT Root}

We try to generalize the map of the previous section. All objects found here will be one-parameter perturbations (with the parameter $c$ ) of the corresponding objects from the previous section. We will refrain from indicating this by an extra $c$ in the notation (to keep it as brief as possible). However, the reader should keep in mind that the unperturbed situation corresponds to $c=0$.

The idea is to stay in the class of symmetric QRT roots of deg $=2$ : in nonhomogeneous coordinates, $g(u, v, 1)=[\widetilde{u}: \widetilde{v}: 1]$ with

$$
\widetilde{u}=v, \quad \widetilde{v}=\frac{\alpha u v+\beta u-1}{u-\alpha v-\beta},
$$

which admit an integral of motion

$$
K(u, v)=\frac{\alpha(\alpha+1)\left(u^{2}+v^{2}-1\right)-(\alpha+1) u v+\beta(u+v)-\beta^{2}}{\left(u^{2}-1\right)\left(v^{2}-1\right)} .
$$


Note that map (26) corresponds to $\alpha=1 / 2, \beta=-1 / 2$. As a characteristic feature we choose the existence of a short singular orbit (the third one in (24)):

$$
\left(q_{8} q_{11}\right) \longrightarrow q_{11} \longrightarrow\left(q_{7} q_{11}\right)
$$

i.e., of a point $q_{11}$ which belongs both to $\mathcal{I}(g)$ and to $\mathcal{I}\left(g^{-1}\right)$. One easily computes:

$$
\begin{array}{r}
\mathcal{I}(g)=\left\{q_{8},\left(1, \frac{1-\beta}{\alpha}\right),\left(-1,-\frac{1+\beta}{\alpha}\right)\right\}, \\
\mathcal{I}\left(g^{-1}\right)=\left\{q_{7},\left(\frac{1-\beta}{\alpha}, 1\right),\left(-\frac{1+\beta}{\alpha},-1\right)\right\},
\end{array}
$$

where

$$
q_{7}=[0: 1: 0], \quad q_{8}=[1: 0: 0] .
$$

We have a one-parameter generalization of the previous case, with

$$
q_{11}=(-1,-1) \in \mathcal{I}(g) \cap \mathcal{I}\left(g^{-1}\right),
$$

under the condition

$$
\alpha=1+\beta
$$

In what follows, we parametrize the coefficients $\alpha, \beta$ according to

$$
\beta=\frac{c-1}{2}, \quad \alpha=\frac{c+1}{2}
$$

Proposition 5 Under condition (31), the map g given in (26) has three confined singular orbits as in (24). Moreover, the point $q_{3}$ is infinitely near to $q_{11}$ (with the slope -1). The map g has a fixed point

$$
q_{9}=\left(\frac{1}{c}, \frac{1}{c}\right) .
$$

The pencil of invariant curves $\{K(u, v)=\lambda\}$ of the map $g$ is as in (23). The eight finite base points $q_{1}, \ldots, q_{6}, q_{10}, q_{11}$ lie on the conic given by the numerator of $K(u, v)$.

Proof The second singular orbit in (24) is confirmed by an easy computation. Let us compute the first (long) singular orbit, starting with the remaining point from $\mathcal{I}\left(g^{-1}\right)$, that is, with

$$
q_{10}=\left(\frac{3-c}{1+c}, 1\right) \in \mathcal{I}\left(g^{-1}\right) .
$$




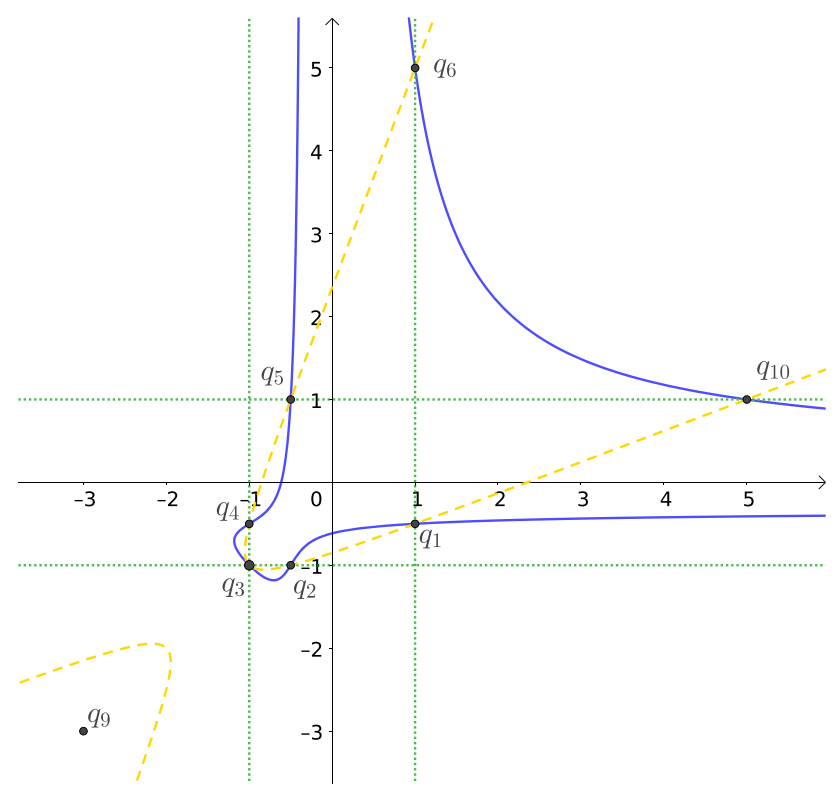

Fig. 3 Geometry of base points of the biquadratic pencil $\{K(u, v)=\lambda\}$ with $K(u, v)$ from (29) with $\beta=-2 / 3, \alpha=1 / 3$, that is, $c=-1 / 3$. All biquadratics pass through $q_{11}=(-1,-1)$ with the slope -1 (corresponding to $q_{3}$ ). Yellow: the conic through the eight base points $q_{1}, \ldots, q_{6}, q_{10}, q_{11}$. Green: four lines $\{u= \pm 1\},\{v= \pm 1\}$

We compute:

$$
\begin{aligned}
& g\left(q_{10}\right)=q_{1}=\left(1,-\frac{1-c}{3+c}\right), \quad g\left(q_{1}\right)=q_{2}=\left(-\frac{1-c}{3+c},-1\right), \\
& g\left(q_{2}\right)=q_{3}>q_{11}=(-1,-1) \text { with slope }-1, \\
& g\left(q_{3}\right)=q_{4}=\left(-1,-\frac{1-c}{3+c}\right), \quad g\left(q_{4}\right)=q_{5}=\left(-\frac{1-c}{3+c}, 1\right), \\
& g\left(q_{5}\right)=q_{6}=\left(1, \frac{3-c}{1+c}\right) \in \mathcal{I}(g) .
\end{aligned}
$$

One easily computes also that $g^{-1}$ blows up the point $q_{10}$ to the line $\left(q_{6} q_{8}\right)$, while $g$ blows up the point $q_{6}$ to the line $\left(q_{7} q_{10}\right)$.

The fixed point $q_{9}$ is given by a straightforward computation (note that for $c \neq 0$, the point $q_{9}$ does not lie on $\left(q_{7} q_{8}\right)$, the line at infinity).

All this is illustrated on Fig. 3.

There holds the following converse to Proposition 2. We perform a quadratic Cremona change of variables based at $q_{9}, q_{10}, q_{11}$ (recall that $q_{9}$ is not a base point of the pencil of invariant curves, while $q_{10}$ and $q_{11}$ are simple base points, the latter having an infinitely close base point $q_{3}$ ).

Proposition 6 Consider a quadratic Cremona map $\phi^{-1}$ blowing down the lines $\left(q_{10} q_{11}\right),\left(q_{9} q_{11}\right),\left(q_{9} q_{10}\right)$ to points denoted by $p_{9}, p_{10}, p_{11}$, respectively, and blow- 
ing up the points $q_{9}, q_{10}, q_{11}$ to the lines $\left(p_{10} p_{11}\right),\left(p_{9} p_{11}\right),\left(p_{9} p_{10}\right)$. All other base points $q_{i}, i=1, \ldots, 8$ are regular points of $\phi^{-1}$ and their images are denoted by $p_{i}=\phi^{-1}\left(q_{i}\right)$. The change of variables $\phi^{-1}$ maps the pencil (23) of biquadratics to the pencil (22) of sextic curves.

Proof The total image of a curve $C$ of the pencil (23) is a curve of degree 8. Since $C$ passes through $q_{10}, q_{11}$, its total image contains the lines $\left(p_{9} p_{11}\right),\left(p_{9} p_{10}\right)$. Dividing by the linear defining polynomials of these two lines, we see that the proper image of $C$ is a curve of degree 6 . This curve passes through all points $p_{i}, i=1, \ldots, 8$ (for $i=7,8$ with multiplicity 2$)$. The curve $C$ of degree 4 intersects the line $\left(q_{10} q_{11}\right)$ at two points $q_{10}, q_{11}$, and two further points, therefore its proper image passes through $p_{9}$ with multiplicity 2 . On the other hand, the curve $C$ of degree 4 has three additional intersection points with each of the lines $\left(q_{9} q_{10}\right)$ and $\left(q_{9} q_{11}\right)$, different from the points $q_{10}$, respectively $q_{11}$. Therefore, its proper image passes through $p_{11}$, resp. $p_{10}$, with multiplicity 3 .

It remains to conjugate the QRT root $g$ by the quadratic change of variables $\phi^{-1}$.

Proposition 7 The map $f=\phi^{-1} \circ g \circ \phi$ is a quadratic Cremona map with three confined singular orbits, as in (21). The eight base points $p_{i}, i=1, \ldots, 6,10,11$ lie on a conic.

Proof We have:

$$
p_{i} \stackrel{\phi}{\longrightarrow} q_{i} \stackrel{g}{\longrightarrow} q_{i+1} \stackrel{\phi^{-1}}{\longrightarrow} p_{i+1}, \quad i=1, \ldots, 5 .
$$

Further,

$$
p_{6} \stackrel{\phi}{\longrightarrow} q_{6} \stackrel{g}{\longrightarrow}\left(q_{7} q_{10}\right) \stackrel{\phi^{-1}}{\longrightarrow}\left(p_{7} p_{10}\right)
$$

(apply Lemma 3 for $\phi^{-1}$ );

$$
\begin{aligned}
& p_{7} \stackrel{\phi}{\longrightarrow} q_{7} \stackrel{g}{\longrightarrow} q_{8} \stackrel{\phi^{-1}}{\longrightarrow} p_{8} ; \\
& p_{8} \stackrel{\phi}{\longrightarrow} q_{8} \stackrel{g}{\longrightarrow}\left(q_{10} q_{11}\right) \stackrel{\phi^{-1}}{\longrightarrow} p_{9} ; \\
& p_{9} \stackrel{\phi}{\longrightarrow}\left(q_{10} q_{11}\right) \stackrel{g}{\longrightarrow}\left(q_{1} q_{10}\right) \stackrel{\phi^{-1}}{\longrightarrow}\left(p_{1} p_{10}\right)
\end{aligned}
$$

(apply Lemma 3 first for $g$, then for $\phi^{-1}$ );

$$
p_{10} \stackrel{\phi}{\longrightarrow}\left(q_{9} q_{11}\right) \stackrel{g}{\longrightarrow}\left(q_{9} q_{10}\right) \stackrel{\phi^{-1}}{\longrightarrow} p_{11}
$$

(apply Lemma 3 for $g$, taking into account that $q_{9}$ is a fixed point);

$$
p_{11} \stackrel{\phi}{\longrightarrow}\left(q_{9} q_{10}\right) \stackrel{g}{\longrightarrow} C\left(q_{9}, q_{1}, q_{10}, q_{7}, q_{11}\right) \stackrel{\phi^{-1}}{\longrightarrow}\left(p_{1} p_{7}\right)
$$


(apply Lemma 3 first for $g$, taking into account that $q_{9}$ is a fixed point and $q_{1}=g\left(q_{10}\right)$; then, the total $\phi^{-1}$-image of the conic is a curve of degree 4 ; however, three lines split off, being the blow-ups of $q_{9}, q_{10}, q_{11}$; thus, the proper image is the line through $\phi^{-1}\left(q_{1}\right)=p_{1}$ and $\left.\phi^{-1}\left(q_{7}\right)=p_{7}\right)$.

Next, we consider lines which are blown down by $f$ :

$$
\left(p_{6} p_{9}\right) \stackrel{\phi}{\longrightarrow}\left(q_{6} q_{9}\right) \stackrel{g}{\longrightarrow}\left(q_{11} q_{9}\right) \stackrel{\phi^{-1}}{\longrightarrow} p_{10}
$$

(apply Lemma 3 first for $\phi$, then for $g$, taking into account that $q_{9}$ is a fixed point);

$$
\left(p_{6} p_{11}\right) \stackrel{\phi}{\longrightarrow}\left(q_{6} q_{11}\right) \stackrel{g}{\longrightarrow} q_{7} \stackrel{\phi^{-1}}{\longrightarrow} p_{7}
$$

(apply Lemma 3 for $\phi$ );

$$
\left(p_{9} p_{11}\right) \stackrel{\phi}{\longrightarrow} q_{10} \stackrel{g}{\longrightarrow} q_{1} \stackrel{\phi^{-1}}{\longrightarrow} p_{1} .
$$

It remains to show that the points $p_{1}, \ldots, p_{6}, p_{10}, p_{11}$ lie on a conic. For this, we observe that the total $\phi^{-1}$-image of the conic $C$ through $q_{1}, \ldots, q_{6}, q_{10}, q_{11}$ is a curve of $\operatorname{deg}=4$, from which two lines split off (blow-ups of $q_{10}, q_{11}$ ). Thus, the proper image is a conic. This conic contains $p_{1}=\phi^{-1}\left(q_{1}\right), \ldots, p_{6}=\phi^{-1}\left(q_{6}\right)$. It also contains $p_{10}$ and $p_{11}$ as the consequence of the fact that $C$ has additional intersection points with both blown-down lines $\left(q_{9} q_{11}\right)$ and $\left(q_{9} q_{10}\right)$, apart from $q_{11}$ and $q_{10}$, respectively.

To make concrete computations, we normalize $\phi^{-1}$ by the following conditions:

$$
p_{9}=(0,1), \quad p_{10}=(-1,0), \quad p_{11}=(1,0),
$$

and

$$
p_{7}=(0,-1)
$$

Then a straightforward computation gives:

$$
\begin{aligned}
\phi(x, y, z)= & \left(1+2 c-c^{2}\right) x y-x^{2}-y^{2}+z^{2}: \\
& 2 c x y-\left(1-c^{2}\right) y z-c^{2} y^{2}-x^{2}+z^{2}: \\
& \left.\left(1+c^{2}\right) x y-c\left(x^{2}+y^{2}-z^{2}\right)\right],
\end{aligned}
$$

and for the further base points given by $p_{i}=\phi^{-1}\left(q_{i}\right)$, we find:

$$
p_{1}=-p_{6}=\left(\frac{(1+c)(3+c)}{5-c^{2}}, \frac{4}{5-c^{2}}\right) \text {, }
$$




$$
\begin{aligned}
& p_{2}=-p_{5}=\left(\frac{(1+c)(3-c)}{(1-c)(3+c)}, \frac{4}{(3+c)(1-c)}\right), \\
& p_{3}=-p_{4}=\left(\frac{3+c}{1-c}, \frac{4}{1-c}\right) \\
& p_{8}=[c: 1: 0] .
\end{aligned}
$$

Theorem 8 The map $f=\phi^{-1} \circ g \circ \phi$ is given by

$$
\begin{aligned}
f=[ & (x-c(y-z))(z-x+(2+c) y): \\
& y((1+c)(z-y)+(3-c) x): \\
& \left.z^{2}+(1-c) x z-(2-c) x^{2}+(1+c)(2-c) x y-y^{2}\right] .
\end{aligned}
$$

In the non-homogeneous coordinates, the map $f(x, y, 1)=[\tilde{x}: \tilde{y}: 1]$ satisfies the following bilinear (Kahan-type) relations:

$$
\left\{\begin{array}{l}
\tilde{x}-x=-(2-c) x \tilde{x}-c(2+c) y \tilde{y}+(1+c)(\tilde{x} y+x \tilde{y})+c, \\
\tilde{y}-y=-(1+c) y \tilde{y}+(\tilde{x} y+x \tilde{y}) .
\end{array}\right.
$$

It possesses an integral of motion

$$
H_{1}(x, y)=\frac{\left(C_{1}(x, y)\right)^{2} C_{2}(x, y)}{\left(1-(x-c y)^{2}\right)\left(1-(x-y)^{2}\right)\left(1-\left(x^{2}+y^{2}-2 c x y\right)\right)},
$$

where

$$
\begin{aligned}
& C_{1}(x, y)=\left(1+c^{2}\right) x y+c\left(1-x^{2}-y^{2}\right), \\
& C_{2}(x, y)=-2\left(1-c-c^{2}\right) x y+\frac{1}{2}\left(3-c-3 c^{2}-c^{3}\right) y^{2}-c x^{2}+c .
\end{aligned}
$$

The base points of the pencil of invariant curves $\left\{H_{1}(x, y)=\lambda\right\}$ are given in (32), (33), and (35)-(38). The conic $\left\{C_{1}(x, y)=0\right\}$ passes through $p_{7}, p_{8}, p_{9}, p_{10}, p_{11}$ (it is the $\phi^{-1}$-image of the line $\left.\left(q_{7} q_{8}\right)\right)$, while the conic $\left\{C_{2}(x, y)=0\right\}$ passes through eight base points $p_{1}, \ldots, p_{6}, p_{10}, p_{11}$.

Proof A straightforward symbolic computation.

On Fig. 4 one can see several invariant curves $\left\{H_{1}(x, y)=\lambda\right\}$ of the map $f$. 


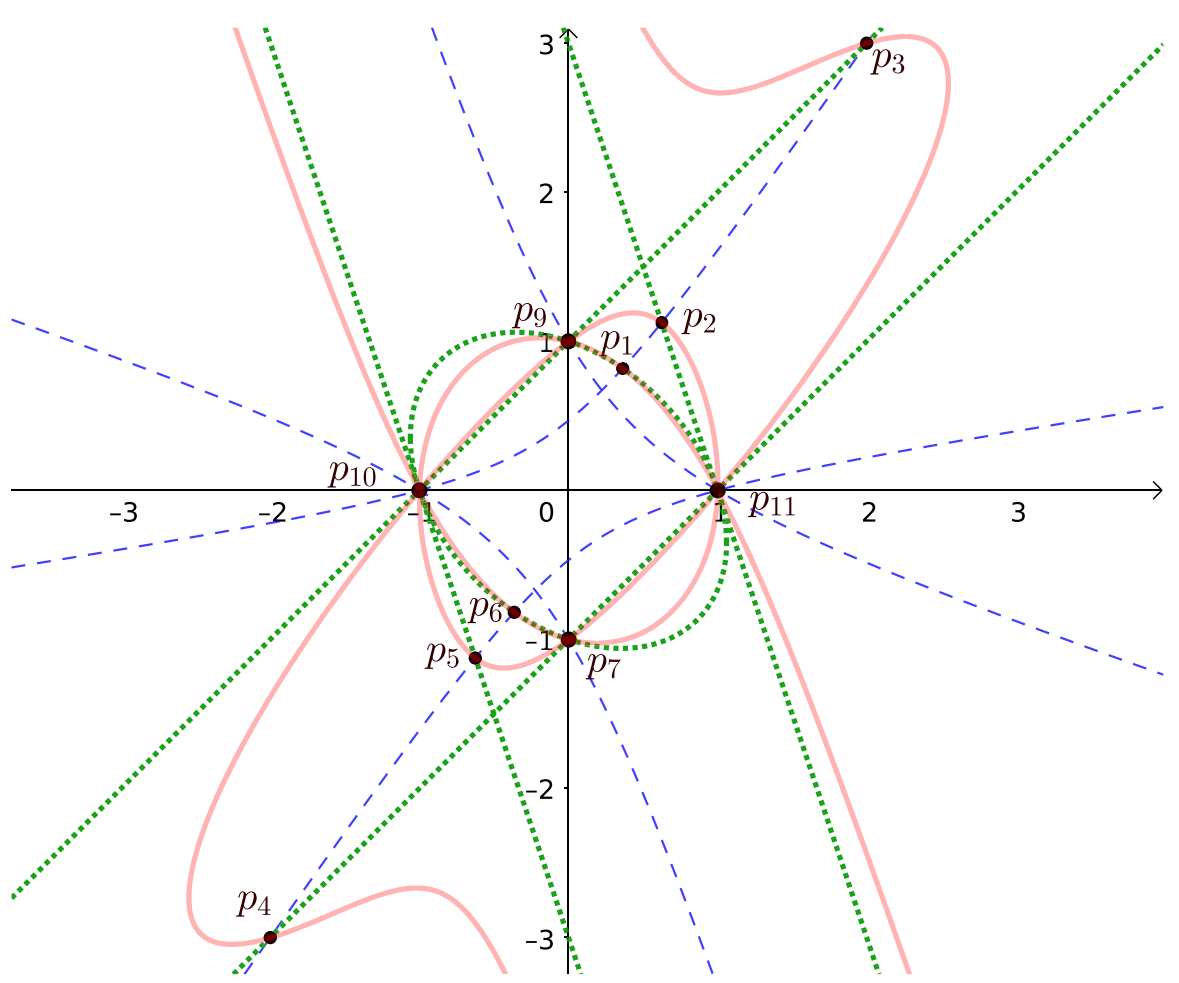

Fig. 4 Some invariant curves of the sextic pencil $H_{1}(x, y)=\lambda$ with $H_{1}(x, y)$ from (41), $c=-1 / 3$. Blue: two conics (one of them with multiplicity 2) - the vanishing set of the numerator of $H_{1}(x, y)$, i.e., $\lambda=0$. Green: four lines and a conic — the vanishing set of the denominator of $H_{1}(x, y)$, i.e., $\lambda=\infty$. Red: a generic curve

\section{Continuous Limit}

Re-scaling $(x, y) \mapsto(\epsilon x, \epsilon y)$ and $c \mapsto \epsilon^{2} c$, we arrive at system (12), which in the limit $\epsilon \rightarrow 0$ is a discretization of system (9). The latter can be written as

$$
\left(\begin{array}{l}
\dot{x} \\
\dot{y}
\end{array}\right)=\frac{1}{(x y+c) y}\left(\begin{array}{c}
\partial H / \partial y \\
-\partial H / \partial x
\end{array}\right)
$$

with

$$
H(x, y)=(x y+c)^{2}\left(-\frac{2}{3} x y+\frac{1}{2} y^{2}+\frac{1}{3} c\right) .
$$

This is a one-parameter (inhomogeneous) perturbation of system (5). Like for the unperturbed system, all level sets $\{H(x, y)=\lambda\}$ of the integral of motion (45) are curves of genus 1 (and of degree 6). Thus, map (12) is a non-trivial integrable Kahantype discretization of (9). 
Integrability of map (12) is in contrast to non-integrability of the straightforward Kahan discretization (11) of (9). We say that singularities of a birational map $f$ of $\mathbb{C P}^{2}$ are not confined, if the orbits of $\mathcal{I}\left(f^{-1}\right)$ are disjoint from $\mathcal{I}(f)$. The non-integrability of maps with non-confined singularities is well studied, see [7]. The better the orbits of $\mathcal{I}\left(f^{-1}\right)$ are separated from $\mathcal{I}(f)$, the stronger non-integrability properties hold.

Proposition 9 The map $f$ generated by bilinear equations (11) is non-integrable, in the sense that its singularities are not confined.

Proof To show this, we restrict ourselves to the case $\epsilon=1$. The resulting quadratic Cremona map has three singularities, $p^{+}=(1,0)$ and two further points not lying on the line $\{y=0\}$. Likewise, the inverse map has three singularities, $p^{-}=(-1,0)$ and two further points not lying on the line $\{y=0\}$. Observe that the line $\{y=0\}$ is invariant. Thus, for the singularities to be confined, we need that some $f^{n}\left(p^{-}\right)=p^{+}$ for some $n \in \mathbb{N}$. The restriction of the map to the line $\{y=0\}$ is given by $\tilde{x}-x=$ $-2 x \tilde{x}+c$, or $\tilde{x}=\varphi(x)=(x+c) /(2 x+1)$. One easily sees that, for a generic $c$, the orbit of $x=-1$ under this Möbius transformation does not hit $x=1$. Indeed, $\varphi^{n}(-1)=1$ is a polynomial equation of degree $n$ for $c$. Thus, for all $c$ but a countable set this equation is not satisfied for any $n \in \mathbb{N}$.

\section{Conclusions}

The results of the present paper confirm that the phenomenon discovered and described in [33] is not isolated, namely that in case of non-integrability of the standard Kahan discretization (when applied to an integrable system), its coefficients can be adjusted to restore integrability. Recall that in the definition of Kahan's discretization the small stepsize $\epsilon$ only appears in the denominator of the differences $(\tilde{x}-x) / \epsilon$ which approximate the derivatives $\dot{x}$, compare (1) and (2). On the contrary, the bilinear expressions on the right hand side of (2) are the exact polarizations of the quadratic vector fields on the right hand side of (1). This discretization method preserves integrability much more frequently than one would expect a priori, but not always. Our examples show that, if the straightforward recipe fails to preserve integrability, certain adjustments of the coefficients by quantities of the magnitude $O\left(\epsilon^{2}\right)$ may allow to restore integrability. Further extending the list of examples and finding their systematic explanation in terms of addition laws on Abelian varieties remains an important and entertaining task for the future. Note that some further examples of discretizations with coefficients depending on the stepsize $\epsilon$ can be found in [22, Sect. 6.5], while an example of a system where polarization yields an integrable discretization in the homogeneous case, but must be adjusted to achieve an integrable discretization in the inhomogeneous case, was given in [34].

Acknowledgements This work was done in the frame of a summer research project of MS and YT at Technische Universität Berlin in the Summer-Fall 2020 (which, due to the pandemic, was performed online). Research of YS is supported by the DFG Collaborative Research Center TRR 109 "Discretization in Geometry and Dynamics".

Funding Open Access funding enabled and organized by Projekt DEAL. 


\section{Declarations}

Conflict of interest The authors declare that there is no conflict of interests.

Open Access This article is licensed under a Creative Commons Attribution 4.0 International License, which permits use, sharing, adaptation, distribution and reproduction in any medium or format, as long as you give appropriate credit to the original author(s) and the source, provide a link to the Creative Commons licence, and indicate if changes were made. The images or other third party material in this article are included in the article's Creative Commons licence, unless indicated otherwise in a credit line to the material. If material is not included in the article's Creative Commons licence and your intended use is not permitted by statutory regulation or exceeds the permitted use, you will need to obtain permission directly from the copyright holder. To view a copy of this licence, visit http://creativecommons.org/licenses/by/4.0/.

\section{References}

1. Bellon, M.P., Viallet, C.-M.: Algebraic entropy. Commun. Math. Phys. 204(2), 425-437 (1999)

2. Celledoni, E., McLachlan, R.I., Owren, B., Quispel, G.R.W.: Geometric properties of Kahan's method. J. Phys. A 46, 025201 (2013)

3. Celledoni, E., McLachlan, R.I., McLaren, D.I., Owren, B., Quispel, G.R.W.: Integrability properties of Kahan's method. J. Phys. A 47, 365202 (2014)

4. Celledoni, E., McLachlan, R.I., McLaren, D.I., Owren, B., Quispel, G.R.W.: Discretization of polynomial vector fields by polarization. Proc. R. Soc. A 471, 20150390 (2015). (10 pp)

5. Celledoni, E., McLachlan, R.I., McLaren, D.I., Owren, B., Quispel, G.R.W.: Two classes of quadratic vector fields for which the Kahan map is integrable. MI Lecture Note, Kyushu University, vol. 74, pp. 60-62 (2016)

6. Celledoni, E., McLaren, D.I., Owren, B., Quispel, G.R.W.: Geometric and integrability properties of Kahan's method: the preservation of certain quadratic integrals. J. Phys. A 52, 065201 (2019)

7. Diller, J.: Dynamics of birational maps of $\mathbb{P}^{2}$. Indiana Univ. Math. J. 45(3), 721-772 (1996)

8. Diller, J.: Cremona transformations, surface automorphisms, and plane cubics. Michigan Math. J. 60, 409-440 (2011)

9. Diller, J., Favre, C.: Dynamics of bimeromorphic maps of surfaces. Am. J. Math. 123(6), 1135-1169 (2001)

10. Duistermaat, J.J.: Discrete Integrable Systems. QRT Maps and Elliptic Surfaces. Springer, New York (2010)

11. Hirota, R.: Nonlinear partial difference equations. I. A difference analogue of the Korteweg-de Vries equation. J. Phys. Soc. Jpn. 43, 1423-1433 (1977)

12. Hirota, R.: Nonlinear partial difference equations. II. Discrete time Toda equation. J. Phys. Soc. Jpn. 43, 2074-2078 (1977)

13. Hirota, R.: Nonlinear partial difference equations. III. Discrete sine-Gordon equation. J. Phys. Soc. Jpn. 43, 2079-2086 (1977)

14. Hirota, R.: Nonlinear partial difference equations. IV. Bäcklund transformation for the discrete-time Toda equation. J. Phys. Soc. Jpn. 45, 321-332 (1978)

15. Hirota, R.: Nonlinear partial difference equations. V. Nonlinear equations reducible to linear equations. J. Phys. Soc. Jpn. 46, 312-319 (1979)

16. Hirota, R.: Discrete analogue of a generalized Toda equation. J. Phys. Soc. Jpn. 50, 3785-3791 (1981)

17. Hirota, R., Kimura, K.: Discretization of the Euler top. J. Phys. Soc. Jpn. 69(3), 627-630 (2000)

18. Hitchin, N.J., Manton, N.S., Murray, M.K.: Symmetric monopoles. Nonlinearity 8(5), 661-692 (1995)

19. Hone, A.N.W., Quispel, G.R.W.: Analogues of Kahan's method for higher order equations of higher degree. In: Nijhoff, F., Shi, Y., Zhang, D. (eds.) Asymptotic, Algebraic and geometric Aspects of Integrable Systems, pp. 175-189. Springer, New York (2020)

20. Kahan, W.: Unconventional numerical methods for trajectory calculations. Unpublished Lecture Notes (1993)

21. Kimura, K., Hirota, R.: Discretization of the Lagrange top. J. Phys. Soc. Jpn. 69(10), 3193-3199 (2000) 
22. Mickens, R.E.: Nonstandard Finite Difference Models of Differential Equations. World Scientific, Singapore (1994)

23. Petrera, M., Pfadler, A., Suris, Yu.B.: On integrability of Hirota-Kimura-type discretizations: experimental study of the discrete Clebsch system. Exp. Math. 18(2), 223-247 (2009)

24. Petrera, M., Pfadler, A., Suris, Y.B.: On integrability of Hirota-Kimura type discretizations. Regular Chaotic Dyn. 16(3-4), 245-289 (2011)

25. Petrera, M., Pfadler, A., Suris, Y.B.: On the construction of elliptic solutions of integrable birational maps. Exp. Math. 26(3), 324-341 (2017). ((with appendix by Y.N. Fedorov))

26. Petrera, M., Suris, Y.B.: On the Hamiltonian structure of Hirota-Kimura discretization of the Euler top. Math. Nachr. 283(11), 1654-1663 (2010)

27. Petrera, M., Suris, Yu.B.: A construction of a large family of commuting pairs of integrable symplectic birational 4-dimensional maps. Proc. R. Soc. A 473, 20160535 (2017). (16 pp)

28. Petrera, M., Suris, Y.B.: New results on integrability of the Kahan-Hirota-Kimura discretizations. In: Euler, N. (ed.) Nonlinear Systems and Their Remarkable Mathematical Structures, pp. 94-120. CRC Press, Boca Raton, FL (2018)

29. Petrera, M., Suris, Y.B.: Geometry of the Kahan discretizations of planar quadratic Hamiltonian systems. II. Systems with a linear Poisson tensor. J. Comput. Dyn. 6, 401-408 (2019)

30. Petrera, M., Smirin, J., Suris, Yu.B.: Geometry of the Kahan discretizations of planar quadratic Hamiltonian systems. Proc. R. Soc. A 475, 20180761 (2019). (13 pp)

31. Petrera, M., Suris, Y.B., Wei, K., Zander, R.: Manin involutions for elliptic pencils and discrete integrable systems. Math. Phys. Anal. Geom. 24, 6 (2021)

32. Petrera, M., Zander, R.: New classes of quadratic vector fields admitting integral-preserving KahanHirota-Kimura discretizations. J. Phys. A Math. Theor. 50, 205203 (2017). (13 pp)

33. Petrera, M., Suris, Y.B., Zander, R.: How one can repair non-integrable Kahan discretizations. J. Phys. A Math. Theor. 53, 37LT01 (2020)

34. Quispel, G.R.W., McLaren, D.I., van der Kamp, P.H.: A novel 8-parameter integrable map in $\mathbb{R}^{4}$. J. Phys. A Math. Theor. 53, 40LT01 (2020)

35. Quispel, G.R.W., Roberts, J.A.G., Thompson, C.J.: Integrable mappings and soliton equations II. Physica D 34, 183-192 (1989)

36. Sanz-Serna, J.M.: An unconventional symplectic integrator of W. Kahan. Appl. Numer. Math. 16, 245-250 (1994)

37. Suris, Y.B.: The Problem of Integrable Discretization: Hamiltonian Approach. Progress in Mathematics, vol. 219. Birkhäuser, Basel (2003)

38. van der Kamp, P.H., Celledoni, E., McLachlan, R.I., McLaren, D.I., Owren, B., Quispel, G.R.W.: Three classes of quadratic vector fields for which the Kahan discretization is the root of a generalised Manin transformation. J. Phys. A Math. Theor. 52, 045204 (2019)

39. van der Kamp, P.H., McLaren, D.I., Quispel, G.R.W.: Generalised Manin transformations and QRT maps. J. Comput. Dyn. 8(2), 183-211 (2021)

40. Zander, R.: On the singularity structure of Kahan discretizations of a class of quadratic vector fields. Eur. J. Math. 7, 1046-1073 (2021)

Publisher's Note Springer Nature remains neutral with regard to jurisdictional claims in published maps and institutional affiliations. 